\section{Los orígenes caribeños del Sistema Nacional de Salud Pública en los EEUU: una aproximación global a la historia de la medicina y de la salud pública en Latinoamérica}

The Caribbean origins of the National Public Health System in the USA: a global approach to the history of medicine and public health in Latin America

\section{Mariola Espinosa}

Profesora, History Department/University of lowa. 280 Schaeffer Hall

52242-1409 - lowa City - IA - EEUU

mariola-espinosa@uiowa.edu

Recebido para publicação em julho de 2013.

Aprovado para publicação em abril de 2014.
ESPINOSA, Mariola. Los orígenes caribeños del Sistema Nacional de Salud Pública en los EEUU: una aproximación global a la historia de la medicina y de la salud pública en Latinoamérica. História, Ciências, Saúde - Manguinhos, Rio de Janeiro, v.22, n.1, jan.-mar. 2015, p.241-253.

\section{Resumen}

Este artículo define la historia global en relación con historia de la medicina y la salud pública. Defiende que una aproximación global a la historia abre un espacio para reverberaciones transmitidas desde la periferia geográfica hacia regiones occidentales, las cuales, tradicionalmente, han dominado la historiografía moderna. Analiza dos intervenciones médicas, en el Caribe, a finales del siglo XIX y principios del $\mathrm{XX}$, y señala que estos sucesos tuvieron profundas consecuencias en los EEUU. Los logros alcanzados en el Caribe, en lo relativo al control de la fiebre amarilla y del anquilostoma, además de servir de modelo para campañas sanitarias en el sur de los EEUU, impulsaron la centralización de la salud pública norteamericana bajo el control centralizador del gobierno federal.

Palabras claves: anquilostoma; fiebre amarilla; historia global; EEUU; Caribe.

\section{Abstract}

This article defines global history in relation to the history of medicine and public health. It argues that a global approach to history opens up a space for examining the reverberations transmitted from the geographic periphery towards western regions, which have traditionally dominated modern historiography. It analyzes two medical interventions in the Caribbean in the late nineteenth and early twentieth century, showing how these events had profound consequences in the USA. The successes achieved in the Caribbean in terms of yellow fever and ancylostoma control, as well as providing a model for health campaigns in the southern USA, inspired the centralization of public health in North America under the centralizing control of the federal government.

Keywords: ancylostoma; yellow fever; global history; USA; Caribbean. 
F Itaban apenas unas horas para el amanecer del 4 de julio de 1905 cuando el buque Tipsey del estado de Mississippi, el cual estaba patrullando las aguas del lago Borgne en la frontera con Luisiana en busca de pescadores italianos que había violado la cuarentena, avistó una pequeña embarcación que intentaba pasar desapercibida. El Tipsey se acercó y, después de ordenar que se detuviera la nave, procedió a abordarla. Lo que los oficiales del Tipsey no se esperaban era que esta barcaza, llamada Tom, fuera parte de las flota del estado de Luisiana y que su tripulación se encontrara escondida. Inmediatamente después de abordar la nave, los tripulantes del Tipsey fueron arrestados por violación de cuarentena y su nave incautada.

Ese mismo día, todas las patrullas del estado de Mississippi encargadas de hacer cumplir la cuarentena fueron obligadas a abandonar el lago Borgne. Una epidemia de fiebre amarilla se había declarado en Nueva Orleans y amenazaba con enturbiar las buenas relaciones existentes entre los dos estados, a los cuales no les faltaban motivos para desconfiar de su respectiva capacidad de controlar la epidemia. Sólo la intervención de las autoridades federales consiguió calmar los ánimos de las autoridades sanitarias de ambos estados (Louisiana..., 5 aug. 1905, p.1). Estos sucesos culminarían, en última instancia, en la centralización del Sistema de Salud Pública bajo el control del gobierno federal de los EEUU. Algunas autoridades sanitarias reclamaban desde mediados del siglo XIX que los asuntos de salud pasaran a manos del gobierno federal de los EEUU. Pero fueron sucesos en Cuba y Puerto Rico los que dieron impulso y contribuyeron a que esta llamada fuese finalmente respondida.

\section{Historia global de la medicina y de la salud pública}

Históricamente, las campañas organizadas por los EEUU para erradicar la fiebre amarilla y el anquilostoma durante los primeros años del siglo XX en el Caribe han sido consideradas como ejemplos de salud pública colonial. Sin embargo, ciertos aspectos de estos sucesos de historia colonial que podrían contribuir a una mayor comprensión de la historia global de la medicina y de la salud pública no han recibido toda la atención que merecen. El estudio de las intervenciones médicas de los EEUU en Cuba y en Puerto Rico ilustra cómo ciertos sucesos que se dan en el Caribe tuvieron un efecto directo en la historia de la medicina y salubridad en los EEUU. La historia global se ocupa no sólo de estas reverberaciones desde la periferia hacia el centro sino también de cómo éstas son percibidas. En Cuba, por ejemplo, el logro de la centralización de la administración de la salud pública por parte del gobierno militar estadounidense sirvió de modelo para la centralización de las iniciativas de control de las epidemias de fiebre amarilla en las ciudades del sur de los EEUU. Del mismo modo, el uso de dispensarios para tratar el anquilostoma en Puerto Rico supuso una fuente de inspiración decisiva en el establecimiento de tratamientos contra el parásito en el sur de los EEUU.

El término historia global se ha popularizado en los últimos años, pero ha servido para describir enfoques históricos diferentes. Como historiadores de América Latina y de la historia de la medicina, debemos manejar una definición clara de la historia global y delinear las diferencias entre éste y otros términos relacionados como, por ejemplo, historia internacional, nacional o colonial. Algunos estudiosos han utilizado el término historia global para referirse a cualquier estudio de temas o acontecimientos que ocurren fuera de los centros hegemónicos; es decir, fuera de EEUU y Europa. Esta definición responde directamente a la 
tradicional distribución de las áreas de estudio dentro de los departamentos de historia de las universidades europeas y estadounidenses, las cuales han establecido la siguiente clasificación: historia americana, historia europea e historia mundial, e incluso, en algunos casos, para esta última se ha utilizado el término "historia de 'otras' partes del mundo". No cabe duda de que se trata de una definición inadecuada ya que el estudio de los acontecimientos históricos de los países de Latinoamérica y de África, la mal llamada periferia, constituyen, en justicia, estudios de historia nacional dentro de sus propios países.

En el área de la historia de la medicina, muchos de los trabajos de este tipo estudian la importancia del desarrollo de las instituciones de salud pública para explicar procesos más generales relacionados con la formación de Estados, la creación de naciones y con procesos de legitimación política. Dentro de dicha área de estudio caben destacar los trabajos de Palmer (2003); Agostoni (2003); Rodríguez (2006); Zulawski (2007); Hochman, Di Liscia, Palmer (2012); y Brotherton (2012). Por otro lado, otros trabajos (ver, por ejemplo, Kropf, 2009; García, 2012) ilustran cómo médicos latinoamericanos, bien inspirados por teorías desarrolladas en otros países, o bien a modo de reacción, desarrollaron tratamientos para enfermedades endémicas de sus propios países. Finalmente, algunos estudios exploran la respuesta de la ciudadanía a la hora de enfrentarse a enfermedades, regulaciones de sanidad y a la disponibilidad de tratamientos (ver por ejemplo, Armus, 2011, publicado en español en 2007). Cualquiera que sea su enfoque, en estos trabajos, los eventos en Europa o Norteamérica sirven principalmente como un plano distante frente al cual la historia nacional se desenvuelve.

También se ha utilizado el término historia global de la medicina para referirse a estudios sobre las organizaciones transnacionales de salud pública. Esta definición es muy limitadora y, por lo tanto, sería más exacto clasificar estos estudios bajo la categoría más amplia de historia transnacional. De esta manera se podrían incluir historias coloniales y neocoloniales que examinan específicamente cómo las ideas médicas y los programas de salud pública, que se originaron en Europa o en los EEUU, fueron transportadas a Latinoamérica. Asimismo, y a diferencia de trabajos anteriores de este tipo, dichos trabajos examinan y evidencian cómo esas ideas y esos programas fueron cuestionados por las poblaciones autóctonas, adaptados a las circunstancias regionales por médicos e incluso propuestos por investigadores locales. ${ }^{1}$

Las extensas actividades de la Fundación Rockefeller en Latinoamérica ofrecen un ejemplo histórico que ha despertado gran interés. Esta organización no logró imponer reformas sanitarias estrictas; por el contrario, sus programas tuvieron éxito solo cuando los oficiales de la fundación negociaron y adaptaron las reformas propuestas para poder dar mejor respuesta a las condiciones y a las preocupaciones de la población local. Entre los trabajos más recientes sobre este tema destacan los de Birn (2006), Faria (2007), Palmer (2010) y Stepan (2012). ${ }^{2}$ Los estudios recientes sobre las reformas médicas borbónicas de finales del siglo XVIII y principios del XIX en Perú y México, y los que se centran en los esfuerzos de los EEUU por controlar enfermedades en Latinoamérica durante la Guerra Fría, son ejemplos de cómo los historiadores han podido documentar y explicar la adopción, adaptación y rechazo de las medidas gestadas fuera de la región. ${ }^{3}$

Se podría afirmar que estos trabajos de investigación forman parte de la historia global: se trata de temas nacionales que se desarrollan fuera del Atlántico Norte, de temas de medicina global por la naturaleza de su alcance, y, finalmente, de temas coloniales y neocoloniales que 
se caracterizan por sus temas inherentemente transnacionales. ${ }^{4}$ Pero la característica distintiva que aporta un enfoque histórico realmente global, parafraseando a Jeremy Adelman (2004, p.408), es que reconoce que los eventos que han ocurrido en el perímetro científico no son necesariamente periféricos a la ciencia.

Entonces lo que hace que un enfoque histórico sea verdaderamente global es que descentraliza el lente mediante el cual estudiamos las historias tradicionales. En la historia de la medicina y de la salud pública, América Latina ha sido frecuentemente concebida como una región en los límites de una pendiente muy empinada, centralizada entorno al mundo del Atlántico Norte; un lugar forzado a tratar con sucesos, conocimiento y prácticas que fluyen inexorablemente cuesta abajo. Tomar un enfoque global es reconocer que la topografía de esta historia, en vez de ser un cono uniforme y estático, es escabrosa y ondulada. Significa también que se pueden identificar las ocasiones en las que las pendientes se han invertido y que, por lo tanto, los que normalmente han estado en la cima se han visto obligados que enfrentarse a los sucesos que descienden hacia América Latina. En otras palabras, el enfoque global sigue el camino marcado por Albert Crosby en su libro The columbian exchange ya que examina no sólo flujos hacia América Latina sino también los que brotan desde la región.

\section{Cuba y Puerto Rico}

Mientras que las medidas de salud pública implementadas por el gobierno de los EEUU en Cuba y Puerto Rico después de la Guerra Hispanoamericana de 1898 tuvieron profundas consecuencias en las islas, los grandes cambios engendrados por dichas experiencias en la administración de la salud pública dentro de los EEUU no han sido reconocidos. Previamente, la salud pública había sido vista en los EEUU como una cuestión estrictamente regional/estatal y, por eso estaba bajo la administración de los Estados y no bajo la del gobierno federal. Estas dos instancias de éxito en el control de enfermedades logrado por las autoridades coloniales de los EEUU en el Caribe - sobre la fiebre amarilla en Cuba y el anquilostoma en Puerto Rico - socavaron los argumentos de que la salud pública sólo era decidida por las condiciones estatales. Por el contrario, como veremos a continuación, estos triunfos aumentaron y finalmente, consolidaron la legitimidad del control federal sobre cuestiones de salud pública.

Para demostrar cuán dramático fue el efecto de estos acontecimientos caribeños en los EEUU, primero vamos a analizar la historia de la salud pública en el siglo XIX, periodo durante el cual el único rol sanitario que jugó el gobierno federal fue el de controlar las cuarentenas internacionales. Luego, discutiremos cómo las experiencias en Cuba y Puerto Rico ayudaron al cirujano general del Servicio Hospital-Marino de los EEUU (US MarineHospital Service, USMHS) Walter Wyman a subrayar la importancia de la creación de una agencia federal de prevención y control de enfermedades. Sugerimos entonces que estos dos ejemplos sirvieron para demostrar que era posible centralizar el control de las enfermedades infecciosas bajo un sistema federal. Las experiencias en Cuba y Puerto Rico fueron modelos para las campañas federales en contra de la fiebre amarilla y el anquilostoma, enfermedades que por décadas habían afligido a los estados del sur de los EEUU.

Durante la mayor parte del siglo XIX, el gobierno federal no ejercía casi ningún poder sobre la salud pública del país; la protección de las enfermedades era responsabilidad de los 
gobiernos estatales. El dominio de los estados en cuestiones de salud pública estaba atado a los conocimientos médicos contemporáneos sobre la transmisión de enfermedades; a principios de siglo se pensaba que éstas eran causadas por miasmas. Las nuevas teorías de bacteriología, a finales de siglo, cambiaron muy poco la manera en la cual las autoridades respondían a problemas de salud pública ya que, al igual que con el miasma, la limpieza era el método de salubridad adecuado para tratar enfermedades y condiciones causadas por infecciones bacterianas. Ya que la recogida de la basura y la limpieza de calles, acueductos y sistemas de alcantarillado protegían solamente los lugares específicos en los cuales estas medidas se implementaban, las competencias en cuestiones de salud pública se delegaban a los gobiernos locales. Por esa razón, y de acuerdo con el conocimiento médico del momento, una política nacional de salud púbica no habría servido un gran propósito.

La idea de que la enfermedad y su prevención eran elementos intrínsecamente locales era tan firme que fue convertida en ley. Frecuentemente, la Corte Suprema de los EEUU utilizaba el ejemplo de la salud pública como el marcador que definía la división de poder dentro del federalismo estadounidense. Si alguna medida pretendía proteger la salud pública o era análoga a una regulación de salubridad, se consideraba competencia de los estados; si no, entonces quizás caía bajo la competencia federal. Sólo entonces Washington podía actuar (Parmet, 1996, p.480). Incluso después de la Guerra Civil, la cual supuso un aumento significativo del poder del gobierno federal, la Corte Suprema reafirmó una y otra vez el poder soberano de los estados en cuestiones de salud pública (Ross, 1998).

De hecho, cualquier intento de incorporar bajo el control federal la salud pública era entendido como contrario al conocimiento médico y vivido como contrario a las instituciones políticas de los EEUU. Por ese motivo, se enfrentaba con una cerrada oposición. Por ejemplo, la Junta Nacional de Salud (US National Board of Health), la cual se creó después de la económicamente desastrosa epidemia de fiebre amarilla de 1878 en la que murieron más de veinte mil personas en una docena de estados del valle del río Mississippi, sólo existió por cuatro años, tiempo suficiente para la publicación de un informe sobre fiebre amarilla en La Habana. En 1882, el congreso de los EEUU decidió cerrarla al cortar su financiación. ${ }^{5}$

A pesar del cierre de la Junta de Salud, la experiencia durante la epidemia de fiebre amarilla de 1878 convenció a algunos oficiales de salud que mantener la salud pública bajo poder estatal era un error. La fiebre amarilla se había extendido siguiendo el cauce del Río Mississippi y las vías ferroviarias, sin respetar las fronteras estatales; de hecho, los primeros casos durante la epidemia fueron de visitantes procedentes de La Habana que habían llegado recientemente a Nueva Orleans. Muchos, incluyendo el cirujano general Wyman, hicieron llamadas para que los EEUU obligara a España a responsabilizarse y controlar la fiebre en Cuba, mientras que otros pedían la anexión de la isla para que los EEUU, por su cuenta, pudiera controlar el problema de una vez por todas (HSMHS, 1896, p.384-439). Al estallar la Guerra de la Independencia Cubana en 1895, el gobierno español envió miles de tropas, no aclimatadas, a áreas de infección de fiebre amarilla y, además, desatendió las condiciones de salubridad en la colonia; las llamadas al gobierno federal para que actuara se multiplicaron. En el otoño de 1897, el gobierno de los EEUU comunicó a los representantes europeos su plan de intervenir en la guerra, en Cuba. La primera justificación fue la amenaza, cada 
vez mayor, a la que se enfrentaba el país, de que una posible epidemia de fiebre amarilla estallaría en la isla y se propagaría a los estados del sur. ${ }^{6}$

No es de extrañar que luego que el ejército norteamericano invadiera la isla y expulsara a los españoles, su prioridad en cuanto a sanidad fuese eliminar la fiebre amarilla, enfermedad que en el país afligía mayormente a extranjeros. Durante los primeros meses de ocupación norteamericana, el gobierno militar comenzó rápidamente a desinfectar las ciudades de la isla. Como los médicos militares confiaban en la creencia de que la fiebre amarilla dependía de la suciedad, sus mayores esfuerzos se concentraron en la higiene. Los trabajadores barrieron las calles, limpiaron a fondo edificios y dragaron la bahía de La Habana depositando los desperdicios en alta mar. Hasta el correo fue fumigado. Sin embargo, la enfermedad continuó cobrando víctimas. ${ }^{7}$

Este fracaso suscitó nuevos esfuerzos para dar con la verdadera causa de la enfermedad. La Comisión Norteamericana de la Fiebre Amarilla, bajo el liderazgo de Walter Reed, comenzó, en 1900, sus trabajos de investigación en las afueras de La Habana. Después de combinar la teoría del médico cubano Carlos J. Finlay, quien argumentaba que solo había una especie de mosquito transmisor de la enfermedad, con las observaciones del médico estadounidense Henry R. Carter, quien había demostrado que pasaban entre diez y 12 días entre la primera infección y las subsiguientes, la Comisión diseñó una serie de experimentos que establecieron, sin lugar a dudas, que la enfermedad solamente se transmitía a través de la picadura del mosquito (Reed, Carroll, Agramonte, 1911 [primera edición de 1901]).

Este avance científico revolucionó los métodos utilizados por el comandante del Ejército Norteamericano encargado de La Habana, William C. Gorgas. A pesar de que Gorgas mantuvo las medidas utilizadas anteriormente para conservar las ciudades limpias, las nuevas políticas de salubridad del gobierno de ocupación se orientaron hacia el exterminio de los mosquitos y de sus criaderos. Las inspecciones sistemáticas de todas las casas de la ciudad dejaron claro que no se toleraría la existencia de receptáculos de agua al descubierto. Este nuevo enfoque fue muy exitoso. En 1900, antes del comienzo de la campaña anti-mosquito, más de 1.200 personas en La Habana habían contraído la fiebre amarilla y 310 murieron a causa de la enfermedad. A finales de 1901, sin embargo, ningún caso fue reportado en la isla (Gorgas, 1901).

El gobierno estadounidense estaba decidido a poner fin a las epidemias de fiebre amarilla en la isla para así proteger a los estados del sur de EEUU de una vez por todas. En 1902, como una de las condiciones para la obtención de la independencia, el nuevo gobierno civil de Cuba fue obligado a incluir en la constitución de la isla el compromiso de mantener las políticas sanitarias establecidas bajo el gobierno de ocupación militar; el no hacerlo podría resultar en una nueva intervención militar. ${ }^{8}$ El gobierno federal - no los gobiernos estatales - ganó la batalla contra la fiebre amarilla que había amenazado a los estados del sur del país durante años.

La eliminación de la fiebre amarilla en Cuba fue un logro sin precedentes de las instituciones de salud pública federales. El mundo quedó asombrado de que La Habana, tristemente conocida como uno de los lugares más insalubres del hemisferio, se transformara casi de la noche a la mañana en una ciudad mucho más limpia y segura que Washington, París, Berlín o Nueva York. ${ }^{9}$

Si bien la eliminación de la amenaza de la fiebre amarilla fue el logro más dramático del gobierno federal de los EEUU en su imperio caribeño, no fue el único. Evidencia adicional de 
la capacidad federal para el control de las enfermedades infecciosas se encuentra en la historia de Puerto Rico, otro de los premios de la guerra de 1898. La anemia endémica que sufrían los puertorriqueños de las áreas rurales había provocado mucho menos preocupación que la fiebre amarilla en La Habana, pero el descubrimiento de su causa tendría consecuencias que se extenderían más allá de las costas de la nueva colonia.

Las tropas estadounidenses que ocuparon Puerto Rico después de la guerra notaron, con sorpresa, el miserable estado en el que se encontraban los campesinos de la isla. La mayoría tenía la ropa hecha andrajos y pocos usaban zapatos. Incluso tomando en consideración su pobreza, la apariencia de los jíbaros era chocante: flacos, pálidos, débiles. En las regiones del sur de los EEUU, donde muchos sufrían síntomas similares, esta condición anémica se atribuía a la desnutrición y al hábito de comer tierra y arcilla. ${ }^{10}$ En Puerto Rico se reportaban muchas muertes a causa de la anemia o de enfermedades relacionadas con la debilidad causada por esta afección. Aproximadamente una tercera parte de todas las muertes en la isla a finales del siglo eran atribuidas a la anemia (Ashford, Gutierrez Igaravidez, 1911, p.8).

Aunque la debilidad de los campesinos puertorriqueños era un tema muy discutido, e incluso tratado con mucho desdén, la anemia no estaba entre las preocupaciones principales de los nuevos gobernadores militares en la isla. En Agosto de 1899, un desastre natural complicó los esfuerzos de consolidar el poder de los EEUU en la antigua colonia española. El huracán San Ciriaco, con un ancho de sesenta millas, y uno de los peores que se han documentado en la isla, cruzó Puerto Rico dejando un sendero de devastación de sureste a noroeste. Muy pocas áreas quedaron indemnes. Por supuesto, como era de esperar, muchas de las casuchas de los campesinos no soportaron ni los ventarrones ni el torrente de lluvia. Además, el huracán destruyó dos terceras partes de las plantaciones de la isla, dejando a la mayor parte de la población rural no sólo sin hogar, sino también sin empleo. En las ciudades, las inundaciones dejaron muchas estructuras inservibles. Al ver la escasez y la creciente desesperación de las víctimas, el ejército norteamericano construyó rápidamente campamentos para suministrar raciones de comida y proporcionar alojamiento. ${ }^{11}$

El teniente Bailey K. Ashford, un joven médico militar, fue puesto al frente de las labores de evacuación de las víctimas del huracán en las afueras de la ciudad de Ponce, al sur de la isla. Muchos de los refugiados padecían de anemia. El conocimiento médico de la época había establecido que la anemia era causada por la desnutrición, por lo tanto, Ashford comenzó a distribuir raciones de carne entre los refugiados, pero no respondieron a esa dieta. Tampoco hubo una mejoría significativa cuando más tarde se distribuyó una dieta más tradicional de arroz, habichuelas y bacalao. De hecho, se reportaron docenas de muertes. Molesto y perplejo por esta epidemia de anemia letal, Ashford comenzó a analizar la sangre de las víctimas del huracán. Las analíticas revelaron síntomas característicos de anquilostomiasis y los posteriores análisis de heces confirmaron el diagnóstico (Ashford, 1900a, 1900b).

El anquilostoma ya había sido tema de intensa investigación científica dos décadas antes, después de una serie de epidemias que habían afectado a los mineros europeos durante las obras de construcción del túnel del Ferrocarril Gotthard, en los Alpes. Ya se sabía, que las larvas del anquilostoma entran en el cuerpo de sus víctimas a través de la piel, preferiblemente a través de la piel que se encuentra entre los dedos de los pies. De ahí pasan, a través del sistema sanguíneo, al intestino delgado. Una vez allí, se adhieren a la pared del intestino 
donde se alimentan de la sangre del enfermo. Es allí también donde, después de desarrollarse, se reproducen y sus huevos salen del cuerpo del enfermo a través de sus deposiciones. Una vez incubados los huevos, el ciclo comienza otra vez. Incluso existía tratamiento: una fuerte dosis de timol para matar a los parásitos, seguida de un laxante de sales de Epsom para eliminar las larvas. El tratamiento se completaba con una segunda dosis. Para prevenir una nueva infección, la persona debía evitar tener contacto a través de la piel con excrementos humanos; es decir, lo único que se requería eran zapatos e instalaciones sanitarias adecuadas. ${ }^{12}$

Antes del descubrimiento de Ashford, la anquilostomiasis apenas se había diagnosticado en los EEUU, excepto en el caso de inmigrantes recientes de Europa. Él estableció que la languidez y la debilidad que los soldados norteamericanos habían observado en Puerto Rico no se debían ni a la desnutrición ni a una inferioridad inherente como se especulaba. Por el contrario, dichos síntomas eran causados por un parásito que estaba directamente relacionado con las condiciones miserables en las cuales vivía la mayor parte de la población en la isla. Menos de uno de cada cinco puertorriqueños usaba zapatos regularmente (Carroll, 1899, p.50-51). En las áreas rurales de la isla, escaseaban los servicios sanitarios básicos; el censo de 1899 había puesto en evidencia que menos de uno de cada tres hogares tenía algún tipo de instalaciones sanitarias (Allen, 1901, p.32).

La economía agrícola, en torno a la cual se desarrollaba la vida de los puertorriqueños del interior de la isla, contribuía también a la persistencia de la anquilostomiasis. Los tres meses de la cosecha de café en las montañas coincidían con la temporada de lluvias y requería un trabajo tan intenso que hombres, mujeres y niños llegaban de toda la isla para cosechar el grano. El suelo fangoso de los cafetales en la época de la cosecha constituía el lugar ideal para el crecimiento de las larvas y la índole del trabajo exigía que el suelo fuerapisado repetidamente por trabajadores descalzos. Al finalizar la cosecha del café, los jornaleros llevaban el parásito en sus cuerpos a otras partes de la isla (Ashford, Gutierrez Igaravidez, 1911, p.11).

No es sorprendente que el descubrimiento de Ashford de las causas reales de la mal llamada anemia endémica fuera la base de la creación de una nueva manera de tratar la enfermedad a gran escala. Primero se comenzó una campaña de educación para que los médicos informaran a los habitantes de la isla de que el anquilostoma era la causa de la anemia común. También les enseñaron a curarla. ${ }^{13}$ Además, Ashford fue puesto a cargo de la nueva Comisión para el Estudio y Tratamiento de la Anemia.

La Comisión tomó un enfoque médico. Ashford y sus colegas produjeron un tratamiento que los puertorriqueños podían administrar por sí mismos. Construyeron pequeños dispensarios y hospitales de campaña, en las áreas rurales de la isla, como el que levantaron en Ponce, para ofrecer servicios médicos a la población. En estos lugares, los campesinos eran diagnosticados de manera oficial y recibían tratamiento para su enfermedad. Poco a poco, las noticias de los beneficios obtenidos gracias al tratamiento comenzaron a diseminarse por la isla y cada vez más personas visitaron los dispensarios (Hill, 1925, p.11). Durante los primeros cinco años de la Comisión, unos trescientos mil puertorriqueños - aproximadamente una tercera parte de la población total de la isla - fueron tratados (Ashford, Gutierrez Igaravidez, 1911, p.34). 


\section{Reverberaciones}

El éxito logrado por el gobierno norteamericano en su nuevo imperio caribeño aumentó la noción de legitimidad de las acciones federales en cuestiones de salud pública. Wyman, el cirujano general del Servicio Hospitalario de la Marina, hacía tiempo que abogaba por incrementar el rol del gobierno federal en este tipo de intervenciones. Después de las intervenciones militares norteamericanas en las dos islas, Wyman consiguió el apoyo político que necesitaba, gracias al control sobre la fiebre amarilla en Cuba y al descubrimiento del anquilostoma en Puerto Rico. ${ }^{14}$ Estos dos casos sirvieron como ejemplos de los beneficios que se podían obtener al centralizar la salud a nivel nacional.

En el 1902 el congreso de los EEUU creó una agencia federal de salud pública, llamada Servicio de Salud Pública Marino-Hospitalario Estadounidense (United States Public Health Marine-Hospital Service, USPHMHS). ${ }^{15}$ El acta de su creación le permitió tener acceso a importantes recursos, como el primer laboratorio federal de investigación, y le otorgó el poder de convocar reuniones entre las autoridades sanitarias estatales una vez al año y, en caso de emergencias sanitarias, cuando fuese necesario. En efecto, la agencia había conseguido el poder de obligar a los estados a actuar bajo las órdenes del gobierno federal (An act..., 1 jul. 1902).

Uno de los primeros trabajos de la ahora fortalecida agencia federal de salud pública fue la investigación del anquilostoma en el sur de los EEUU. El investigador principal fue Charles Wardell Stiles del Departamento de Agricultura, quien dos años antes concluyó que los especímenes puertorriqueños enviados por Ashford a su laboratorio para su diagnóstico eran una especie de anquilostoma distinta a la que ya había sido identificada en Europa.

Inicialmente, Stiles visitó las regiones rurales de los estados de Virgina, Carolina del Norte y Carolina del Sur, donde encontró evidencia extensa de la existencia de anquilostoma en fincas y plantaciones en las cuales el suelo era arenoso y no de arcilla y denunció que el parásito era el responsable real de "la proverbial vagancia de los blancos pobres" del sur (Stiles, 1902). Más tarde hallaría que el anquilostoma también se encontraba en los estados de Georgia y de Florida. Siguiendo el ejemplo de Ashford en Puerto Rico, Wyman ordenó que Stiles educara a los médicos del sur sobre la enfermedad y así Stiles comenzó una gira por las escuelas de medicina de la región. Sin embargo, la construcción de dispensarios, siguiendo el modelo puertorriqueño de Ashford, tendría que esperar hasta 1909, año en el que Nelson Rockefeller fundó la organización que más tarde se convertiría en la Fundación Rockefeller.

El brote de fiebre amarilla de julio de 1905 en Nueva Orleans supuso la primera prueba de aceptación, por parte de las autoridades estatales, de las competencias federales en cuestiones de salud pública. Las autoridades estatales se movilizaron rápidamente y pusieron en marcha las mismas medidas que habían sido tan efectivas en La Habana: drenaron, cubrieron con aceite o rejilla cualquier receptáculo que pudiera ser criadero de mosquitos y aislaron a los casos sospechosos de haber contraído la fiebre amarilla. A pesar de eso, dos semanas más tarde, y con el pánico escalando entre la población, las autoridades sanitarias estatales se reunieron con el alcalde de Nueva Orleans y con el gobernador del estado de Luisiana para implorar al presidente Theodore Roosevelt que pusiera al USPHMHS a la cabeza de la campaña que doblegaría la enfermedad (Louisiana..., 5 aug. 1905, p.1). 
Las autoridades sanitarias federales que llegaron a Nueva Orleans no trajeron nuevas técnicas: simplemente continuaron aplicando las ya exitosamente probadas medidas de control del mosquito. Tampoco trajeron dinero federal: el estado de Louisiana y los ciudadanos de Nueva Orleans aportaron los fondos para el financiamiento de la campaña sanitaria. Lo que las autoridades federales sí brindaron fue legitimidad. Anteriormente, las autoridades sanitarias de distintas jurisdicciones se habían enzarzado en discusiones, a veces violentas, que cuestionaban la eficacia de sus respectivos esfuerzos. Por ejemplo, ese mismo día, mientras las autoridades locales pedían ayuda federal, los barcos de cuarentena de Mississippi, que patrullaban el lago Borgne, eran detenidos a punto de pistola en el ataque furtivo perpetrado por la reserva naval de Luisiana. La tripulación fue acusada de piratería y, subsecuentemente, detenida (Louisiana..., 5 aug. 1905, p.1).

La capacidad de liderazgo demostrada por el gobierno federal contribuyó, definitivamente, a restaurar el orden en una situación que rápidamente caía fuera de control. Al fin y al cabo, en momentos de emergencia sanitaria, los gobiernos estatales y locales no confiaban en que otros estados implementaran eficazmente las medidas necesarias de salud pública, pero sí confiaban en el gobierno federal. Como indicaba el telegrama del gobernador de Mississippi a su homólogo en Luisiana, después de retirar la reserva militar que había sido enviada a la frontera, luego de los sucesos en el lago Borgne, "si el barco patrulla Grace, del estado de Mississippi, hubiera estado al servicio del Departamento Hospitalario de los EEUU, no lo habría podido secuestrar. No estoy luchando contra el Gobierno Federal" (Archbishop..., 6 aug. 1905, p.1).

Esa confianza estaba bien fundamentada. El líder de la campaña federal, doctor Joseph H. White, había aportado una gran eficiencia a los esfuerzos sanitarios del gobierno federal. Bajo su dirección, la enfermedad no se propagó fuera de Nueva Orleans y los últimos casos de fiebre amarilla ocurrieron seis semanas antes de la llegada del invierno, lo que llevó a la auto extinción de la epidemia. Los sucesos fueron elogiados mundialmente no sólo como prueba irrefutable de la teoría de la transmisión de la enfermedad por la picadura del mosquito, establecida en La Habana, sino también como una demostración definitiva de que el gobierno federal era superior a los estados a la hora de enfrentarse de manera eficiente y definitiva a las epidemias.

En este trabajo he detallado dos breves ejemplos de cómo el acercamiento desde la historia global de la medicina ilumina aspectos hasta ahora ignorados de los esfuerzos de la salud pública colonial norteamericana en el Caribe. La historia global de la medicina y de la salud pública es más estrecha que la historia de la medicina en la frontera global y más extensa que la historia de organizaciones globales de la salud. Verla como una historia que cuenta cómo los sucesos ocurridos en la periferia desencadenan cambios en los países del centro hegemónico, nos permite documentar y poner de manifiesto procesos transnacionales, pero lo hace sin oscurecer la desigualdad existente en las relaciones entre los distintos países. A la vez, esta definición permite entablar un diálogo entre trabajos académicos en el campo de historia de la medicina y de la salud pública y las tendencias académicas que buscan "descentralizar" la historia global y, al hacerlo, demuestra que las experiencias imperiales en las colonias fueron esenciales a las historias de los países de la metrópoli. Además de las profundas consecuencias que 
estos sucesos tuvieron para Cuba y Puerto Rico, los esfuerzos contra la fiebre amarilla y la anquilostomiasis ayudaron a legitimar una nueva y más extensa función del gobierno federal de los EEUU en cuestiones de salud pública, algo que hasta ese momento había sido considerado como paradigmáticamente fuera del alcance del poder federal.

\section{NOTAS}

${ }^{1}$ Confieso que no me siento completamente cómoda con el uso de los términos "colonial/neocolonial" ya que no todas las relaciones de desigualdad entre los países estudiados en este tipo de trabajo son lo suficientemente desequilibradas como para constituir realmente casos de colonialismo o neocolonialismo. Sin embargo, este término es más útil, en mi opinión, que otros términos alternativos como "internacional", que corren el riesgo de oscurecer por completo el verdadero elemento de desigualdad que reside en el corazón de estas relaciones.

2 Para una discusión sobre el uso del término "salud global" como sustituto de "salud internacional", ver Brown, Cueto, Fee (2006).

${ }^{3}$ Para las reformas Borbónicas en el Perú y en el sur de México, ver, respectivamente, Warren (2010) y Ramírez (2012). Para los programas anti-malaria en México durante la Guerra Fría, ver Cueto (2007).

${ }^{4}$ Sara Hodges (2012) critica la tendencia a utilizar el término "global" para describir una gran cantidad de trabajos de historia de la medicina.

${ }^{5}$ Para más información sobre la creación de la Junta y la resistencia en contra de ésta, ver Humphreys (1992, p.13-14, 63-64) y Ellis (1992, p.60-82).

${ }^{6}$ Stewart L. Woodford to Secretary of State John Sherman, September 13, 1897; October 4, 1897 and October 5, 1897 (USDS, 1901, p.562-565, 573-579); ver, también, Espinosa (2006, p.541-568).

${ }^{7}$ Para más información sobre los primeros pasos tomados por el ejército norteamericano para combatir la fiebre amarilla, ver Espinosa (2009, capítulo 3).

${ }^{8}$ Article V, Platt Amendment (USA, 1901, p.897-898); Acta de la Convención Constituyente, June 12, 1901 (República..., 1918, p.591-593).

${ }^{9}$ Uno de muchos ejemplos de este punto de vista se puede ver en Topics... (26 oct. 1901).

${ }^{10}$ Ver, por ejemplo, Pitt (feb.-apr. 1808, p.340-341).

${ }^{11}$ Para más información sobre los estragos causados por el huracán San Ciriaco y cómo respondió el gobierno militar norteamericano en la isla, ver Schwartz (1992).

${ }^{12}$ Para más información sobre los trabajos de erradicación y prevención del anquilostoma en el noreste de Italia, Egipto y Brasil, ver Palmer (2010, p.22-34).

${ }^{13}$ Ver, por ejemplo, Porto... (1899).

${ }^{14}$ Ver carta de Walter Wyman a George Vest (Wyman, 3 feb. 1902).

${ }^{15}$ La agencia fue rebautizada, en 1912, con el nombre de Servicio de Salud Pública de Estados Unidos (United States Public Health Service).

\section{REFERENCIAS}

AN ACT...

An act to increase the efficiency and change the name of the United States Marine Hospital Service, 32 Stat., 712. 1 jul. 1902.

ADELMAN, Jeremy.

Latin American and world histories: old and new approaches to the Pluribus and the Unum. Hispanic American Historical Review, v.84, n.3, p.399-410. 2004.
AGOSTONI, Claudia.

Monuments of progress: modernization and public health in Mexico City, 1876-1910. Calgary:

University of Calgary Press. 2003.

ALLEN, Charles H.

First annual report of the governor of Porto Rico. Washington: Government Printing Office. 1901. 
ARCHBISHOP...

Archbishop Chapelle yellow fever patient: federal officers plan to control situation at New Orleans. New York Times, p.1. 6 aug. 1905.

ARMUS, Diego.

The ailing city: health, tuberculosis, and culture in Buenos Aires, 1870-1950. Durham: Duke University Press. 2011.

ARMUS, Diego.

La ciudad impura: salud, tuberculosis y cultura en Buenos Aires, 1870-1950. Buenos Aires: Edhasa. 2007.

ASHFORD, Bailey K.

Anklyostomiasis in Puerto Rico. Journal of the American Medical Association, v.34, p.1057. 1900a.

ASHFORD, Bailey K.

Anklyostomiasis in Puerto Rico. New York Medical Journal, v.71, p.552-556. 1900b.

ASHFORD, Bailey K.; GUTIERREZ IGARAVIDEZ, Pedro.

Uncinariasis (Hookworm Disease) in Porto Rico: a medical and economic problem. Washington, D.C.: Government Printing Office. 1911.

BIRN, Anne-Emanuelle.

Marriage of convenience: Rockefeller international health and revolutionary Mexico. Rochester: University of Rochester Press. 2006.

BROTHERTON, P. Sean.

Revolutionary medicine: health and the body in post-soviet Cuba. Durham: Duke University Press. 2012.

BROWN, Theodore; CUETO, Marcos; FEE, Elizabeth.

A transição de saúde pública "internacional" para "global" e a Organização Mundial da Saúde. História, Ciências, Saúde - Manguinhos, v.13, n.3, p.623-647. 2006.

CARROLL, Henry K.

Report on the island of Porto Rico. Washington: Government Printing Office. 1899.

CUETO, Marcos.

Cold War, deadly fevers: malaria eradication in Mexico, 1955-1975. Washington: Woodrow Wilson Center Press. 2007.

ELLIS, John H.

Yellow fever and public health in the New South. Lexington: University Press of Kentucky. 1992.

ESPINOSA, Mariola.

Epidemic invasions: yellow fever and the limits of Cuban independence, 1878-1930. Chicago: University of Chicago Press. 2009.

ESPINOSA, Mariola.

The threat from Havana: southern public health, yellow fever, and U.S. intervention in the Cuban struggle for independence, 1878-1898. Journal of Southern History, v.72, n.3, p.541-568. 2006.

FARIA, Lina.

Saúde e política: a Fundação Rockefeller e seus parceiros em São Paulo. Rio de Janeiro: Editora Fiocruz. 2007.

GARCÍA, Mónica.

Producing knowledge about tropical fevers in the Andes: preventive inoculations and yellow fever in Colombia, 1880-1890. Social History of Medicine, Oxford, v.25, n.4, p.830-847. 2012.

GORGAS, William C.

Report of vital statistics of the City of Havana, year 1901, file 1900/275. Records of the Military Government of Cuba, record group 140, entry 3, box 135. (National Archives, College Park, MD). 1901.

HILL, Rolla B.

The uncinariasis problem. Porto Rico Health Review, v.1, n.1, p.11. 1925.

HOCHMAN, Gilberto; DI LISCIA, María Silvia; PALMER, Steven (Ed.).

Patologías de la patria: enfermedades, enfermos y nación en América Latina. Buenos Aires: Editorial Lugar. 2012.

HODGES, Sara.

The global menace. Social History of Medicine, v.25, n.3, p.719-728. 2012.

HUMPHREYS, Margaret. Yellow fever and the South. Baltimore: Johns Hopkins University Press. 1992.

KROPF, Simone Petraglia.

Carlos Chagas e os debates e controvérsias sobre a doença do Brasil. História, Ciências, Saúde Manguinhos, v.16, supl.1, p.205-227. 2009.

LOUISIANA...

Louisiana asks nation to fight yellow fever. New York Times, p.1. 5 aug. 1905.

PALMER, Steven.

From popular medicine to medical populism: doctors, healers, and public power in Costa Rica, 1800-1940. Durham: Duke University Press. 2003.

PALMER, Steven.

Launching global health: the Caribbean odyssey of the Rockefeller Foundation. Ann Arbor: University of Michigan Press. 2010.

PARMET, Wendy E.

From Slaughter-House to Lochner: the rise and fall of the constitutionalization of public health. American Journal of Legal History, v.40, n.4, p.476505. 1996. 
PITT, Joseph.

Observations on the country and diseases near Roanoke River, in the state of North Carolina. New York Medical Reports, 2nd Series, v.5, p.340341. feb.-abr. 1808.

PORTO....

Porto Rico Superior Board of Health. Circular n.5: Anemia - Its cause, treatment, and prevention. San Juan: A. Lynn e hijos de Pérez Moris. 1899.

RAMÍREZ, Paul.

"Like Herod's massacre": quarantines, Bourbon reform, and popular protest in Oaxaca's smallpox epidemic, 1796-1797. The Americas, v.69, n.2, p.203-235. 2012.

REED, Walter; CARROLL, James; AGRAMONTE, Arístides.

The etiology of yellow fever: an additional note. In: Yellow fever: a compilation of various publications. Washington: Government Printing Office. 1911.

REPÚBLICA...

República de Cuba. Senado. Memoria de los trabajos realizados durante las cuatro legislaturas y sesión extraordinaria del primer período congresional, 1902-1904. Habana: Imprenta y Papelería de Rambla, Bouza. 1918.

RODRÍGUEZ, Julia.

Civilizing Argentina: science, medicine and the modern state. Chapel Hill: University of North Carolina Press. 2006.

ROSS, Michael A.

Justice Miller's reconstruction: The SlaughterHouse Cases, health codes, and civil rights in New Orleans, 1861-1873. Journal of Southern History, v.64, n.4, p.649-676. 1998.

SCHWARTZ, Stuart B.

The hurricane of San Ciriaco: disaster, politics, and society in Puerto Rico, 1899-1901. Hispanic American Historical Review, v.72, n.3, p.303-334. 1992.

STEPAN, Nancy Leys.

The national and the international in public health: Carlos Chagas and the Rockefeller Foundation in Brazil, 1917-1930s. Hispanic American Historical Review, v.91, n.3, p.469-502. 2012.
STILES, Charles Wardell.

Hookworm disease in the South. Frequency of infection by the parasite (Uncinaria americana) in rural districts. Preliminary report to the Surgeon General. US Public Health and MarineHospital Service. Public Health Reports, v.17, n.43, p.2433-2434. 1902.

TOPICS...

Topics of the times. New York Times, p.8. 26 oct. 1901.

USA.

United States of America. The statutes at large of the United States of America. v.31. Washington: Government Printing Office. 1901.

USDS.

United States Department of State. Papers relating to the foreign relations of the United States, with the annual message of the president transmitted to congress, December 5, 1898. Washington: Government Printing Office. 1901.

USMHS.

United States Marine-Hospital Service. Annual report of the supervising surgeon-general of the Marine-Hospital Service of the United States for the fiscal year 1895. Washington: Government Printing Office. 1896.

WYMAN, Walter.

Walter Wyman to George Vest, Chairman Committee on Public Health and National Quarantine, US Senate, feb. 3, 1902, 57th Congress, 1st Session. House of Representatives, report n.2415. 3 feb. 1902.

WARREN, Adam.

Medicine and politics in colonial Peru: population growth and the Bourbon Reforms. Pittsburgh: University of Pittsburgh Press. 2010.

ZULAWSKI, Ann.

Unequal cures: public health and political change in Bolivia, 1900-1950. Durham: Duke University Press. 2007. 